\title{
Desigualdad de género en el sistema penitenciario de Bahía y su impacto en la resocialización de las mujeres encarceladas
}

\author{
Gender Inequality in the Bahia Penitentiary System and Its Impact on the \\ Resocialization of Incarcerated Women
}

\section{Desigualdade de gênero no sistema prisional da Bahia e seu impacto na ressocialização de mulheres encarceradas}

Sandro José Gomes

Centro de Estudios e Investigaciones para el Desarrollo Docente CENID A.C., México sandro.gomes@seap.ba.gov.br https://orcid.org/0000-0001-7637-8809

\section{Resumen}

El propósito de esta investigación fue analizar la posición desigual vivida por las mujeres en el sistema penitenciario de Bahía, Brasil, y el impacto de dicha posición en la resocialización de la prisión. La investigación cualitativa se realizó con base en una revisión bibliográfica actual sobre el tema e incluyó, en el plan concreto de la investigación, el análisis documental de cinco informes de entidades que dan seguimiento al encarcelamiento de personas en el estado de Bahía y el respeto de los derechos humanos en estas cárceles. Los resultados muestran que, de un total de 25 unidades penitenciarias, solo nueve hacen custodia de mujeres y la mayoría de las unidades no tienen las condiciones mínimas para ejercer esta labor. Por lo tanto, el presente artículo destaca que el sistema penitenciario de Bahía no está preparado para recibir mujeres y, cuando lo hace, les da un trato aún peor que el que reciben los hombres, lo que hace imposible la posibilidad de la resocialización de la prisión.

Palabras clave: cuestiones de género, encarcelamiento femenino, rehabilitación penitenciaria. 


\section{Revista Iberoamericana \\ de las Ciencias Sociales y \\ Humanísticas}

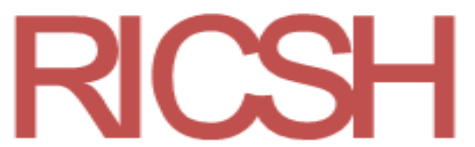

ISSN: $2395-7972$

\section{Abstract}

The purpose of this paper is to analyze the unequal position assumed by women in the Bahia prison system and the impact of this position on prison resocialization. The qualitative research was carried out based on a current bibliographic review on the subject and had, in the specific research plan, the documentary analysis of five reports of entities that follow up on the issues of imprisonment of people in the state of Bahia, Brazil, and respect for human rights in these prisons. The survey results show that, out of a total of 25 prison units, only nine have custody of women and most of the units do not have the minimum conditions to carry out this work. Thus, the present article shows that the penitentiary system of Bahia is not prepared to receive women and when it does it gives them even worse treatment than that given to men, making the prospect of prison resocialization impossible.

Keywords: female incarceration, gender issues, penitentiary rehabilitation.

\section{Resumo}

O objetivo desta pesquisa foi analisar a posição desigual assumida pelas mulheres no sistema penitenciário da Bahia, Brasil, e o impacto dessa posição na ressocialização da prisão. A pesquisa qualitativa foi realizada com base em uma revisão bibliográfica atual sobre o assunto e continha, no plano concreto da investigação, a análise documental de cinco relatórios de entidades que acompanham as questões de encarceramento de pessoas e respeito pelos direitos humanos nas prisões da Bahia. Os resultados da investigação mostram que, de um total de 25 unidades penitenciárias, apenas nove mantinham a custódia de mulheres e a maioria delas não possui condições mínimas para vigiar as mulheres. Portanto, este artigo destacou que o sistema penitenciário da Bahia não está preparado para receber mulheres e, quando o faz, oferece a elas um tratamento ainda pior do que o recebido pelos homens, o que impossibilita a ressocialização das mulheres presas.

Palavras-chave: encarceramento feminino, questões de gênero, reabilitação penitenciária.

Fecha Recepción: Julio 2019

Fecha Aceptación: Diciembre 2019 


\section{Revista Iberoamericana \\ de las Ciencias Sociales y \\ Humanísticas}

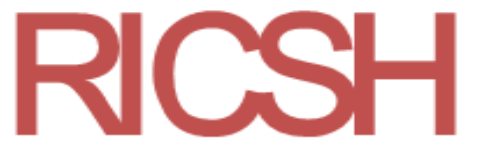

ISSN: $2395-7972$

\section{Introducción}

Este artículo tiene como objetivo analizar la posición desigual asumida por las mujeres en el sistema penitenciario de Bahía, Brasil, y el impacto de esta posición en la resocialización de la prisión.

En el levantamiento de las preguntas de investigación se ha tenido en cuenta que la prisión es una institución total, por lo tanto, está constituida como un sistema sociocultural que tiene sus reglas, normas, procedimientos, valores, castigos, sanciones y creencias, y la supervivencia física, moral e intelectual de los encarcelados depende completamente de su adaptación a este sistema que se construyó dentro de un contexto social de dominación masculina total (Almeda y Di Nella, 2017; Lobo y Vilarrubia, 2017; Santoro, Pereira y Lara, 2018).

También se ha tenido en cuenta que la ley brasileña, Ley Federal N 7210/1984 (Brasil, 2009) establece que el propósito de las penas de prisión no es solo castigar a los reclusos, sino también darles condiciones para que puedan reintegrarse efectivamente a la sociedad, es decir, resocializar (Gomes y Silva, 2017); sin embargo, en la práctica se nota, a lo largo del país, un predominio de la defensa del castigo en detrimento de la adopción de políticas resocializadoras (Pekny y de Mattos, 2017), lo cual se convierte en un obstáculo para la rehabilitación de personas encarceladas.

La literatura internacional muestra que en las cárceles donde el enfoque en la rehabilitación es mayor que el castigo las relaciones de los prisioneros con los trabajadores de la prisión mejoran (Lambert, Barton y Hogan, 2014). Tal es la importancia que debe tener la resocialización del preso que Gomes (2016) argumenta que los administradores de la prisión deben tratar de comprender las características organizativas que favorezcan la rehabilitación de los presos.

Debe mencionarse, en este sentido, que la ley brasileña entiende que es función del Estado aplicar medidas políticas socioeducativas para mejorar la condición social del individuo destinado a cumplir la pena privativa de libertad; por lo tanto, se considera a la resocialización como el buen uso de los programas aplicados al preso a través de la custodia, con especial énfasis en la asistencia sanitaria, social, educativa y laboral (Gomes, 2016; Gomes y Silva, 2017).

Finalmente, se ha tenido en cuenta que la ley brasileña aún no aborda las cuestiones de género que impregnan el ambiente carcelario, ni cómo tales relaciones pueden afectar la resocialización de la prisión; la única categoría legítima para diferenciar el conjunto de personas 


\section{Revista Iberoamericana \\ de las Ciencias Sociales y \\ Humanísticas}

ISSN: $2395-7972$

encarceladas es la sexual, ya que en la prisión todo se mezcla menos los sexos (Almeida, 2018; Barbosa de Souza y Zini, 2015).

La justificación de esta investigación apunta a la necesidad de un estudio más profundo de la presencia de mujeres en las cárceles, debido al creciente crimen femenino y la ineficacia de los procesos punitivos actuales del Estado brasileño, el cual no puede regresar al prisionero a la sociedad debidamente rehabilitado del crimen. Respecto al lugar de la investigación, el sistema penitenciario de Bahía fue el elegido debido a que las cárceles de esta entidad, según Gomes (2016), son bastante representativas del sistema penitenciario brasileño y tienen las mismas deficiencias que se encuentran en las de otros estados del país.

En este contexto, es pertinente interpretar la prisión femenina desde una perspectiva de género en el sistema penitenciario de Bahía, porque, a causa de la gran cantidad de prisioneros varones, la situación extremadamente vulnerable de las mujeres encarceladas es invisible, por lo tanto, la investigación tuvo como punto de partida el siguiente problema: ¿en qué medida las cuestiones de género en el sistema penitenciario de Bahía afectan la resocialización de las prisiones?

\section{Metodología}

La investigación cualitativa se realizó con base en una revisión bibliográfica actual sobre el tema y tuvo como protagonista, en el plan concreto de la investigación, el análisis documental. En este sentido, según Ana y Lemos (2018), la investigación documental tiene al documento como objeto de investigación y se puede utilizar como fuente de información, indicaciones y aclaraciones que aportan su contenido para dilucidar ciertos problemas y servir de prueba para otros, de acuerdo con el interés del investigador. Así, la investigación documental se caracteriza por la búsqueda de información en documentos que no han recibido ningún tratamiento científico, por ejemplo, los informes, lo que permite producir o reelaborar el conocimiento y crear nuevas formas de comprender fenómenos, interpretar hechos, sintetizar información, determinar tendencias y, en la medida de lo posible, hacer inferencias, todo lo cual justifica el uso de este tipo de investigación para producir nuevos conocimientos en investigación social (Ana y Lemos, 2018; Cascaes y Rosa, 2018; De Andrade, Schmitt, Storck, Piccoli y Ruoff, 2018).

Por lo tanto, el análisis de documentos recurre, al mismo tiempo, a la técnica de recolección y análisis de datos y método de investigación (De Andrade et al., 2018). En el presente estudio, el 


\section{Revista Iberoamericana \\ de las Ciencias Sociales y \\ Humanísticas}

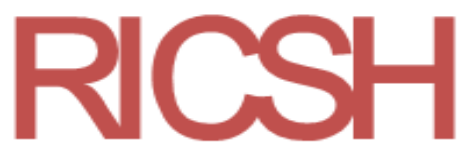

ISSN: $2395-7972$

análisis documental se utilizó como una técnica para el procesamiento de datos, con el objetivo de transformar la información, con el propósito de hacer que sea más comprensible y capaz de establecer correlación con otros datos de fuentes secundarias.

Así, fueron analizados, en octubre de 2019, los siguientes informes:

a) Plano Estadual de Atenção à Mulher Privada de Liberdade e Egressas do Sistema Prisional 2018-2019. Presenta un diagnóstico del sistema penitenciario para mujeres en Bahía, caracterizando todas las nueve unidades penitenciarias que custodian a mujeres.

b) Relatório Visita Aos Presídios. Proporciona datos sobre la inspección de las unidades penitenciarias en la capital, incluido el Conjunto Penal de Mujeres, y aborda aspectos relacionados con la resocialización de los reclusos, como el trabajo interno, actividades deportivas y culturales, actividades educativas, atención médica, infraestructura, alimentos, etc.

c) Relatório das audiências de custódias na comarca de Salvador/BA: anos de 2015-2018. Partiendo de los datos obtenidos en las audiencias de custodia, aporta datos sobre el perfil de los presos y presas que ingresan al sistema penitenciario en la capital de Bahía.

d) Relatório Sobre o Conjunto Penal de Feira de Santana. Se trata de un diagnóstico con respecto a la infraestructura, aspectos relacionados con la seguridad y la resocialización de los reclusos y reclusas en el Conjunto Penal de Feira de Santana, con especial énfasis en el pabellón de mujeres.

e) Relatório Sobre o Conjunto Penal de Paulo Afonso. Un diagnóstico sobre infraestructura, seguridad y resocialización de prisioneros y prisioneras en el Conjunto Penal Paulo Afonso, destacando el pabellón de mujeres.

A continuación, en la tabla 1 , se muestran los métodos de recolección de datos de los informes. 


\section{Revista Iberoamericana \\ de las Ciencias Sociales y \\ Humanísticas}

Tabla 1. Métodos de recolección de datos

\begin{tabular}{|c|c|}
\hline & todos de recolección de datos \\
\hline $\begin{array}{l}\text { Plano Estadual de } \\
\text { Atenção à Mulher } \\
\text { Privada de Liberdade } \\
\text { e Egressas do Sistema } \\
\text { Prisional 2018-2019 }\end{array}$ & $\begin{array}{l}\text { Los datos fueron recolectados entre } 2014 \text { y } 2017 \text { por funcionarios de } \\
\text { prisiones del Gobierno de Bahía en unidades que custodian a mujeres } \\
\text { a través de la observación directa, entrevistas con reclusos y } \\
\text { trabajadores de prisiones, y se realizó un análisis de los registros } \\
\text { médicos de los prisioneros y otros documentos de registro y control de } \\
\text { prisioneros. El documento fue producido por la Secretaría de } \\
\text { Administración Penitenciaria [SEAP] (2018). }\end{array}$ \\
\hline $\begin{array}{l}\text { Relatório Visita Aos } \\
\text { Presídios }\end{array}$ & $\begin{array}{l}\text { Los datos fueron recolectados en } 2016 \text { en el Conjunto Penal Femenina } \\
\text { por la Comisión Especial del Sistema Penitenciario de la Orden de } \\
\text { Abogados de Brasil }[\mathrm{OAB}] \text { (2016), Sección de Bahía, mediante } \\
\text { observación directa y entrevistas con trabajadores penitenciarios. }\end{array}$ \\
\hline $\begin{array}{l}\text { Relatório das } \\
\text { audiências de } \\
\text { custódias na comarca } \\
\text { de Salvador/BA: anos } \\
\text { de 2015-2018 }\end{array}$ & $\begin{array}{l}\text { La recolección de datos se llevó a cabo entre } 2015 \text { y } 2018 \text { por la } \\
\text { Especializada Criminal y de Ejecución Penal de la Defensoría Pública } \\
\text { del Estado de Bahía [Dpeba] (2019) en el Centro Penitenciario en } \\
\text { Flagrante, antes de que las prisioneras fueran llevadas a las unidades } \\
\text { penitenciarias de la capital a través del análisis de documentos de las } \\
\text { audiencias de custodia. }\end{array}$ \\
\hline $\begin{array}{l}\text { Relatório Sobre o } \\
\text { Conjunto Penal de } \\
\text { Feira de Santana }\end{array}$ & $\begin{array}{l}\text { Los datos fueron recolectados en } 2018 \text { en el Conjunto Penal de Feira } \\
\text { de Santana por el Sindicato de Trabajadores Penitenciarios del Estado } \\
\text { de Bahía [Sinspeb] (2018) por medio de la observación directa y } \\
\text { entrevistas con trabajadores penitenciarios y prisioneros. }\end{array}$ \\
\hline $\begin{array}{l}\text { Relatório Sobre o } \\
\text { Conjunto Penal de } \\
\text { Paulo Afonso }\end{array}$ & $\begin{array}{l}\text { Los datos fueron recolectados en } 2019 \text { en el Conjunto Penal de Paulo } \\
\text { Afonso también por el Sinspeb (2019) a través de la observación } \\
\text { directa y entrevistas con trabajadores penitenciarios y prisioneros. }\end{array}$ \\
\hline
\end{tabular}

Fuente: Elaboración propia

Dichos informes están disponibles en la sede de las respectivas instituciones o en los sitios web institucionales de la Secretaría de Administración Penitenciaria [SEAP] (2018), de la Orden de Abogados de Brasil [OAB] (2016), de la Defensoría Pública del Estado de Bahía [Dpeba] (2019) y del Sindicato de Trabajadores Penitenciarios del Estado de Bahía [Sinspeb] (2018, 2019), que 


\section{Revista Iberoamericana \\ de las Ciencias Sociales y \\ Humanísticas}

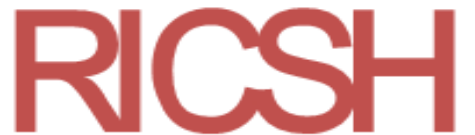

ISSN: $2395-7972$

son organismos que dan seguimiento a los temas sobre el encarcelamiento de personas en la entidad en cuestión y el respeto de los derechos humanos en estas cárceles.

\section{Resultados}

Actualmente, en Bahía hay un total de 25 unidades penitenciarias, de las cuales solo nueve hacen custodia de mujeres. En la capital, Salvador, existe la única unidad exclusivamente femenina del estado, el Conjunto Penal Femenino, y el Hospital de Custodia y Tratamiento, que, además de hombres, también custodia a mujeres con trastornos mentales en conflicto con la ley. Las otras unidades están ubicadas en el interior del estado en las siguientes ciudades: Feira de Santana, Itabuna, Juazeiro, Vitória da Conquista, Teixeira de Freitas, Jequié y Paulo Afonso, totalizando siete establecimientos penitenciarios mixtos, llamados conjuntos penales, que hacen la custodia de hombres y mujeres ya condenadas y también provisionales (SEAP, 2018).

Considerando la alta tasa de encarcelamiento en Brasil, el número de prisioneros es mayor que el número de lugares en las cárceles, pues la tasa de ocupación promedio en las cárceles brasileñas es de 167 \% (Boiteux, 2017), hecho que también es una realidad en Bahía (Gomes, 2016; Sinspeb, 2018).

Así, la existencia de 25 unidades penitenciarias es aún insuficiente para satisfacer la realidad del sistema penitenciario estatal, porque la Bahía tiene la cuarta población más grande del país, es el quinto estado en tamaño, en términos de extensión territorial es más grande que muchos países de Europa, como Francia, por ejemplo; además, es la entidad que genera más divisas con otros estados de Brasil (un total de ocho divisas): Sergipe, Alagoas, Pernambuco, Piauí, Tocantins, Goiás, Minas Gerais y Espírito Santo, algunos de los cuales lideran la clasificación del crimen de Brasil (Gomes, 2016).

\section{Realidad de las mujeres encarceladas en Bahía según el informe de la SEAP}

Con respecto a la situación de las mujeres en las cárceles femeninas, como lo señala el Plano Estadual de Atenção à Mulher Privada de Liberdade e Egressas do Sistema Prisional 20182019 (SEAP, 2018): 


\section{Revista Iberoamericana de las Ciencias Sociales y Humanísticas}

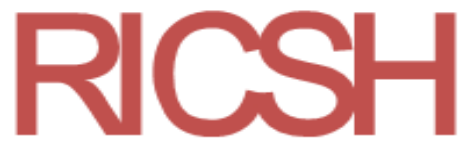

ISSN: $2395-7972$

Se observa que la situación general de las unidades penitenciarias bahianas que custodian a las mujeres no difiere de las unidades de otros estados brasileños, comenzando con la estructura física. Aunque algunas tienen un módulo específico para alojar a las mujeres, carecen de adaptaciones a las especificidades de las mujeres, ya que son el resultado de improvisar espacios destinados previamente para otros fines (por ejemplo, custodia masculina, unidades de seguridad, enfermería, etc.). Solo una tiene una cafetería (Conjunto Penal Femenino), solo una tiene una guardería (Conjunto Penal de Feira de Santana) (...). En todas las unidades (excepto el Hospital de Custodia y Tratamiento), a menudo las mujeres embarazadas ocupan camas, cuyo acceso se vuelve riesgoso debido a la altura y la falta de soporte. Antes de que sean enviadas al arresto domiciliario, las mujeres con bebés a menudo comparten células comunes y viven en un ambiente poco saludable y, a veces, promiscuo (pp. 20-21).

El sistema penitenciario de Bahía, con respecto a la custodia de mujeres, así como otros sistemas penitenciarios del Brasil, aunque encarcela mujeres en espacios centrados específicamente en la prisión femenina, según lo que establece la Ley Federal $N^{\circ} 7210 / 1984$; sin embargo, como se decía y tal y como lo explicaron Santoro et al. (2018), las detenidas aún están sujetas a la idea de que el criminal es casi exclusivamente hombre y, por lo tanto, la formulación de los espacios en las cárceles son proporcionales a esta demanda, es decir, prácticamente orientadas a los hombres; aspecto presentado en el Plano Estadual de Atenção à Mulher Privada de Liberdade e Egressas do Sistema Prisional 2018-2019.

Esta ausencia de espacios que tengan en cuenta el género subraya el hecho de que las políticas penitenciarias de las mujeres no son proporcionales al crecimiento del encarcelamiento femenino. En este sentido, según Boiteux (2017), aunque el número de mujeres detenidas en Brasil es significativamente menor que el masculino en términos absolutos (actualmente 33793 o $5.8 \%$ del total), debe ser tenido en consideración que la tasa de crecimiento del encarcelamiento femenino es incluso mayor que la de los hombres. De hecho, de acuerdo con los datos obtenidos en la Encuesta Nacional de Información Penitenciaria, Infopen Mujeres (Ministério da Justiça e da Segurança Pública, 2016), Brasil ocupó el tercer lugar en la lista de países con el mayor número de mujeres en la cárcel del mundo, en comparación con la tasa de 100000 habitantes, por encima incluso de Rusia y China, países con una población mayor que la brasileña. 


\section{Revista Iberoamericana \\ de las Ciencias Sociales y \\ Humanísticas}

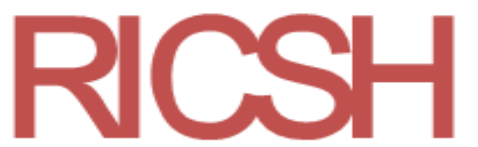

ISSN: $2395-7972$

Aun con todo, dado que las mujeres representan una pequeña porción de la población carcelaria en comparación con la población masculina, son tratadas con indiferencia e inferioridad y no disfrutan equitativamente de la atención brindada a los hombres, lo que hace que el proceso de resocialización sea aún más complejo para las mujeres (Santoro et al., 2018).

Además de esto, debe mencionarse que si bien la prisión para mujeres, tanto en Brasil como en otros países latinoamericanos, expresa y revela claramente las desigualdades de género presentes en diferentes espacios sociales, estas ganan una mayor proporción en el entorno penitenciario por la falta de condiciones para auxiliar a las presas (Almeda y Di Nella, 2017); un ejemplo es la maternidad de la mujer encarcelada, que se convierte en una causa de doble sufrimiento (Laier, 2016), conforme apunta el informe del Gobierno del estado de Bahía hecho por la SEAP (2018), que reconoce esta realidad en sus cárceles.

Los datos señalados por el propio Gobierno de Bahía son ratificados por los datos aportados por la OAB (2016), por la Dpeba (2019) y por el Sinspeb (2018, 2019).

\section{Realidad de las mujeres encarceladas en Bahía señalada por el informe de visita a las prisiones producido por la $\mathrm{OAB}$}

La Comisión Especial del Sistema Penitenciario y Seguridad Pública de la OAB (2016) menciona en su informe que las condiciones del Conjunto Penal Femenino en Salvador se evalúan como regulares; pero las reclusas no tienen actividades deportivas, el trabajo interno es escaso y el número de agentes penitenciarias es insuficiente.

\section{Realidad de las mujeres encarceladas en Bahía señalada por el informe de la Dpeba}

En cuanto a las causas del arresto de mujeres que llegan al Conjunto Penal Femenino, el informe preparado por la Dpeba (2019) señala lo siguiente:

Las mujeres representan un total de solo 1025 de un total de 17793 en el período de septiembre de 2015 a diciembre de 2018. De este total, 862 mujeres se declaran negras y solo 28 se declaran blancas. En términos porcentuales, las mujeres negras representan $96.9 \%$ del total flagrante en el período. En cuanto a las decisiones tomadas, en relación con las mujeres, el porcentaje de libertades provisionales otorgadas fue de $61.6 \%$. El número de detenciones preventivas decretadas, a su vez, 


\section{Revista Iberoamericana \\ de las Ciencias Sociales y \\ Humanísticas}

ISSN: 2395 - 7972

representa un porcentaje de $27.4 \%$. Con respecto a la edad, es posible notar que la mayoría se concentraron en el grupo de edad joven (total de $59.1 \%$ hasta 29 años). Las mujeres arrestadas en flagrantes de septiembre de 2015 a diciembre de 2018, por lo tanto, son predominantemente negras $(96.9 \%)$ y jóvenes $(59.1 \%)$. Finalmente, en relación con las imputaciones penales sufridas por las mujeres entrevistadas, se registraron 483 casos de delitos contra la propiedad (47.1\% del total); 398 casos de ley de drogas ( $38.8 \%$ del total) y 125 casos de otros delitos o el $12.2 \%$ del total (pp. 101- 103).

En Bahía, como en otros estados de Brasil, como en otros países latinoamericanos, el arresto de mujeres es un problema social (Darke y Karam, 2017; Lobo y Vilarrubia, 2017; Santoro et al., 2018), perspectiva que se corrobora con los datos presentados por la Dpeba (2019), que muestran que los delitos más cometidos por las mujeres son los delitos asociados con su situación financiera, como los delitos contra la propiedad (principalmente el hurto y el robo) y el tráfico de drogas, principalmente por mujeres jóvenes que buscan recursos financieros.

En Bahía, especialmente en la capital, la pobreza también se asocia con problemas raciales, teniendo en cuenta que, en el periodo señalado en la anterior cita, $96.9 \%$ de las prisioneras eran negras, perfil que, según De Andrade y Ferreira (2014), caracteriza a la población carcelaria predominante en las cárceles de Brasil. Si el delito de robo se separa del delito de hurto, la mayoría de las mujeres alojadas en las cárceles de Bahía fueron detenidas por el delito de tráfico de drogas, $49.5 \%$ (SEAP, 2018), es decir, el tráfico de drogas aumentó el porcentaje de encarcelamiento femenino en comparación con el porcentaje masculino.

En este contexto, debe mencionarse la relación entre género y criminalidad señalada por la literatura especializada (Almeida, 2018; Lobo y Vilarrubia, 2017; Santoro et al., 2018), que muestra que las mujeres entran en actividades criminales como el narcotráfico como subordinadas a la participación de los hombres en este tipo de delito. Así, Almeida (2018) señala que el crimen femenino se debe a sus relaciones afectivas y los hombres son los principales motivadores para la entrada de las mujeres en el crimen. 


\section{Revista Iberoamericana \\ de las Ciencias Sociales y \\ Humanísticas}

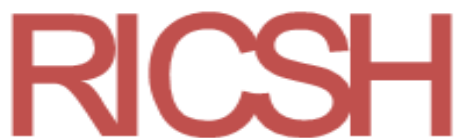

ISSN: $2395-7972$

\section{Realidad de las mujeres encarceladas en Bahía señalada por los informes del Sinspeb}

En el interior de Bahía, informes del Sinspeb $(2018,2019)$ registran las deficiencias de los pabellones femeninos de las siete unidades penitenciarias, con especial énfasis en el establecimiento penal más grande del interior, el Conjunto Penal de Feira de Santana, que, en el informe producido en 2018, se consideró inapropiado para custodiar mujeres. Aunque hubo una reforma y expansión del pabellón masculino, el pabellón de mujeres no se incluyó. Así, en este establecimiento penitenciario, las mujeres son alojadas en "un edificio viejo, maltratado, improvisado, desguazado y poco saludable (...). El pabellón de mujeres [es] un espacio muy inhumano" (Sinspeb, 2018, p. 7).

Además, el informe del Sinspeb producido en 2019 señala que en el Conjunto Penal de Paulo Afonso, una de las unidades penitenciarias más alejadas de la capital y una de las más superpobladas del estado de Bahía, las mujeres arrestadas están en un pabellón totalmente insalubre, no hay una unidad materno-infantil, no hay enfermería para mujeres, no hay biblioteca y sufren de la falta de dentista (al menos durante un periodo mayor a un año); a veces incluso carecen de artículos de higiene personal para ellas. Muchas mujeres ya están en el régimen semiabierto y, por lo tanto, deberían trabajar fuera de la prisión, pero esto no sucede, solo los hombres del régimen semiabierto tienen trabajo fuera de la prisión. Y con respecto a los estudios, solo hay escuela primaria, de manera que quien necesita de escuela secundaria no tiene cómo estudiar (Sinspeb, 2019).

Según los informes del Sinspeb $(2018,2019)$, en muchas de las cárceles de custodia de mujeres no hay agentes penitenciarias suficientes, falta actividades deportivas, escasez de atención médica y exámenes médicos, falta de cuidado dental, el trabajo interno es escaso, la estructura física no es saludable y las actividades educativas son limitadas, aspectos que, de acuerdo con esta misma fuente, son un obstáculo para la resocialización. 


\section{Revista Iberoamericana \\ de las Ciencias Sociales y \\ Humanísticas}

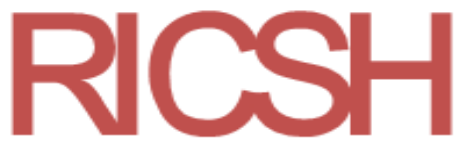

ISSN: $2395-7972$

\section{Discusión}

Los datos de los cinco informes analizados muestran que el sistema penitenciario de Bahía con respecto al encarcelamiento femenino es bastante representativo del sistema penitenciario de Brasil, ya que, de acuerdo con la revisión de la literatura, uno y otro presentan problemas similares: En ciertas situaciones, el sistema penitenciario brasileño trata a las mujeres tal como trata a los hombres. Esto significa que el estado no recuerda que necesitan de papel higiénico cada vez que van al baño, exámenes ginecológicos (pruebas de Papanicolaou), exámenes prenatales durante el embarazo y tampones. Muchas de estas mujeres, por ejemplo, recogen migajas de pan para usar cuando están menstruando. La lucha diaria de estas mujeres es por la higiene y la dignidad. Las mujeres arrestadas son vistas como peores que los hombres que cometen crímenes, ya que en una sociedad aún patriarcal y sexista se impone a las mujeres conductas que no contrastan con la idea de la naturaleza femenina (Santoro et al., 2018, p. 92).

De igual forma, Dos Santos (2018) sostiene que, aunque en Brasil existe la Política Nacional de Atención a la Mujer, que se creó tratando de modificar la realidad de la prisión femenina, los datos presentados cada año por la Encuesta Nacional de Información Penitenciaria muestran que en la práctica esta política no se ha aplicado con efectividad en ningún momento en ningún estado de Brasil; porque la mentalidad de que las mujeres se insertan en una realidad diferente a la de los hombres aún no se ha creado en la sociedad y desde el Gobierno brasileño, y particularmente en el Gobierno del estado de Bahía (Sinspeb, 2018), se insiste en adaptar para las mujeres lo que se creó para los hombres.

Por lo tanto, el análisis de estos informes juntos muestra que la mayoría de las mujeres llegó a la unidad penitenciaria por tráfico y formación de pandillas, si bien no ejercieron el liderazgo en la delincuencia, por lo que los directores de la prisión y los agentes penitenciarios no tienen el mismo cuidado con las mujeres, en términos de seguridad, que necesitan tener con los hombres y las colocan en lugares que ya no son apropiados para estos. Es notable, en este sentido, que también hay una diferenciación en el tratamiento que se dispensa a mujeres arrestadas y hombres arrestados, porque los "frentes", los que dan órdenes en la prisión, quienes cuando hablan son escuchados por la dirección, los agentes penitenciarios, el equipo de salud y todos en la prisión, generalmente son hombres; por otro lado, una mujer solo recibe el mismo trato que los hombres cuando es una presa 


\section{Revista Iberoamericana \\ de las Ciencias Sociales y \\ Humanísticas}

ISSN: $2395-7972$

peligrosa, generalmente la que ordena el tráfico en algún lugar y desempeña un papel que los varones juegan en su pabellón (Zamboni, 2017).

Otro aspecto que debe considerarse en relación con las cárceles de Bahía es el hecho de que la mayoría de sus gerentes son varones y oficiales de la Policía Militar, cuyo modelo de gestión no se centra en las personas y, según Gomes (2016) y Sinspeb (2019), dan poca importancia a la resocialización de los prisioneros y no respetan las peculiaridades de género.

En esa línea, los informes destacan lo que ponen en su documento Santoro et al. (2018), quienes sostienen que a las mujeres se les asigna lo que queda del sistema penitenciario masculino; así, las cárceles que ya no sirven para albergar delincuentes masculinos están destinadas a las mujeres, también los recursos asignados al sistema penitenciario se destinan principalmente a las cárceles de hombres y la atención de la administración de las unidades penitenciarias se centra más en estos.

Además, no puede dejar de mencionarse que la omisión de la más importante ley penal brasileña (Ley Federal N 7210/1984) en relación con el género, según Almeida (2018), es motivo de preocupación, porque no es posible disociar las cuestiones de género de comportamientos y actitudes que ocurren dentro de la prisión (Laier, 2016; Serrano, 2016).

Consecuentemente, debido a la omisión de la ley penal y la presencia de una política nacional de atención a la mujer ineficiente, asociada con las limitaciones de la administración penitenciaria del Gobierno de Bahía con respecto a las mujeres encarceladas, debe ser apuntado que en el sistema penitenciario bahiano no se reconocen las peculiaridades de género de las reclusas y, por esto, tales mujeres están sujetas a un tratamiento que disminuye en mayor medida estas características, lo que afecta su resocialización. Y aún más, según lo que se encontró en los informes y se discutió en la literatura del área, no habrá perspectivas de mejoras significativas en las condiciones actuales de las mujeres encarceladas en dicha unidad federativa mientras se ignoren las peculiaridades de género.

En cuanto a las dificultades del presente estudio, hay que decir que a pesar de la dificultad de encontrar estudios similares sobre el sistema penitenciario en Bahía, lo que implica en la escasez de referencias y en vista de las limitaciones de los informes, considerando que un informe puede ser rebatido con otros informes; en esta investigación, los datos acerca de las condiciones de privación de libertad de las mujeres señalados en cada informe analizado fueron complementarios entre sí y con la investigación bibliográfica, aunque producidos por instituciones que a veces presentan intereses antagónicos como el Gobierno del Estado y el Sindicato de 


\section{Revista Iberoamericana \\ de las Ciencias Sociales y \\ Humanísticas}

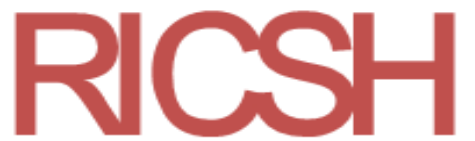

ISSN: $2395-7972$

Trabajadores de Prisiones. Así, la correlación entre los datos presentados en cada informe, cuya metodología de recopilación de datos para cada informe se describe en la Tabla 01, debe presentarse como uno de los puntos fuertes de esta investigación.

\section{Conclusiones}

En conclusión, los resultados presentados y discutidos en los subtítulos anteriores indican que el sistema penitenciario de Bahía, especialmente en el interior del estado, no está preparado para recibir mujeres y, cuando lo hace, le da un trato aún peor que el que se le da a los hombres, lo que impide la posibilidad de resocialización propugnada en la legislación penitenciaria brasileña.

A partir de los informes investigados sobre las condiciones de privación de libertad de las mujeres en las unidades penales bahianas, es posible decir que la mayoría de las mujeres encarceladas ahí son negras, pobres y jóvenes, y los delitos más cometidos son los asociados con cuestiones financieras, como el hurto, el robo y el tráfico de drogas. Estas mujeres son colocadas en unidades penitenciarias que omiten las especificidades de género, ya que son el resultado de improvisar espacios destinados previamente para otros fines. En general, se trata de entornos muy inhumanos para custodiar personas. Por lo tanto, en la mayoría de las unidades con mujeres encarceladas en Bahía el ambiente es promiscuo y poco saludable, especialmente para las embarazadas.

Aunado a lo anterior, en casi todas la unidades penitenciarias las reclusas no tienen actividades deportivas, las actividades educativas son limitadas, hay deficiencia en el cuidado de la salud de las reclusas, el trabajo interno es escaso y el número de agentes penitenciarias es insuficiente; situación que se agrava por el hecho de que la mayoría de los gerentes de las unidades penitenciarias que hacen la custodia de mujeres son varones y oficiales de la Policía Militar, cuyo modelo de gestión no se preocupa de la resocialización de las prisioneras y no respetan sus peculiaridades de género.

Por lo tanto, las cuestiones de género en el sistema penitenciario de Bahía afectan la resocialización de las mujeres encarceladas hasta el punto de hacer inviables las actividades de reinserción que deberían ser parte de la rutina de la prisión; aspecto que hace factible la inferencia de la escasez de políticas penitenciarias específicas para las mujeres que funcionen en el estado. 


\section{Revista Iberoamericana \\ de las Ciencias Sociales y \\ Humanísticas}

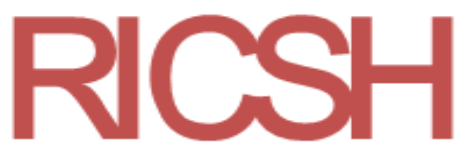

ISSN: 2395 - 7972

Respecto a las limitaciones del presente estudio, debe mencionarse que consultar solo cinco documentos puede no cubrir muchos otros aspectos importantes que arrojen luz respecto a las peculiaridades de género en la prisión. Así, debe considerarse que en algunos casos el número de documentos no permite hacer inferencias; además, los informes son muestras no representativas de los fenómenos estudiados que pueden no traducir la información real, ya que a veces no se prepararon con el fin de proporcionar datos para una investigación posterior; pero, aunque se reconocen las limitaciones de esta investigación, esta podrá servir como base para futuros estudios sobre temas de género en el sistema penitenciario de Bahía.

En este sentido, se recomienda la continuidad de esta investigación utilizando métodos que combinen enfoques cualitativos y cuantitativos como el discurso del sujeto colectivo (DSC), que en Brasil es una metodología ampliamente utilizada en tesis de doctorado y disertaciones de maestría y en investigaciones publicadas en revistas científicas en las áreas de salud, educación, seguridad pública y sistema penal; una técnica que condensa en un único discurso informaciones obtenidas de diversos testimonios, teniendo como producto final una opinión colectiva que refleja los pensamientos, representaciones, creencias y valores de este colectivo sobre un tema dado, ampliando la mirada ante los hallazgos en los informes sobre las condiciones de privación de libertad de las mujeres en el estado de Bahía.

Así, se espera que esta investigación proporcione subsidios científicos que puedan contribuir a la comprensión de las especificidades de las mujeres en un sistema centrado exclusivamente en las necesidades de los hombres. 


\section{Revista Iberoamericana \\ de las Ciencias Sociales y \\ Humanísticas}

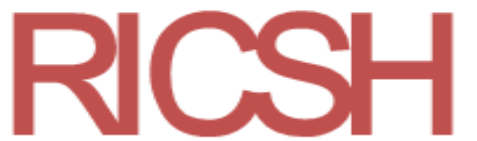

ISSN: $2395-7972$

\section{Referencias}

Almeda, E. y Di Nella, D. (2017). Mujeres y cárceles en América Latina: perspectivas críticas y feministas. Papers: Revista de Sociología, 102(2), 183-214.

Almeida, G. M. (2018). As invisíveis do cárcere: interfaces identitárias de mulheres aprisionadas. (tesis de maestría). Universidade Federal de Sergipe, São Cristóvão.

Ana, W. P. S. e Lemos, G. C. (2018). Metodologia Científica: a pesquisa qualitativa nas visões de Lüdke e André. Revista Eletrônica Científica Ensino Interdisciplinar, 4(12), 531-541.

Barbosa de Souza, M. e Zini, O. (2015). Identidade de gênero no sistema prisional brasileiro. Seminário Internacional Demandas Sociais e Políticas Públicas na Sociedade Contemporânea. UNISC, Santa Cruz do Sul. Recuperado de https://online.unisc.br/acadnet/anais/index.php/sidspp/article/viewFile/13222/2266.

Boiteux, L. (2017). Brasil: Las cárceles de la droga y de la miseria. Nueva Sociedad, 268(2) 14-22. Brasil (2009). Lei n. 7.210, de 11 de julho de 1984: institui a Lei de Execução Penal, Brasília, Brasil: Câmara dos Deputados. Edições Câmara. Recuperado de http://bd.camara.gov.br/bd/handle/bdcamara/766\#main-content.

Cascaes, I. F. e Rosa, A. P. K. (2018). Pesquisa Científica: Uma Breve Abordagem. MaiêuticaArte e Cultura, 6(1), 73-78.

Darke, S. y Karam, M. L. (2017). Las prisiones de América Latina. Ecuador Debate, (101), 53-71.

De Andrade, S. R., Schmitt, M. D., Storck, B. C., Piccoli, T. e Ruoff, A. B. (2018). Análise documental nas teses de enfermagem: técnica de coleta de dados e método de pesquisa. Cogitare Enfermagem, 23(1).

De Andrade, U. S. e Ferreira, F. F. (2014). Crise no sistema penitenciário brasileiro: capitalismo, desigualdade social e prisão. Revista Psicologia, Diversidade e Saúde, 2(1), 24-38.

Defensoria Pública do Estado da Bahia [Dpeba]. (2019). Relatório das audiências de custódias na comarca de Salvador/BA: anos de 2015-2018. Salvador, Brasil: Escola Superior da Defensoria Pública do Estado da Bahia. Recuperado de http://www.defensoria.ba.def.br/wp-content/uploads/2019/09/relatorio-audiencia-decustodia.pdf.

Dos Santos, R. L. (2018). Do Cárcere ao Trabalho: A reinserção de ex-presidiárias no mercado de trabalho. Pesquisa \& Debate, 2(54), 103-115. 


\section{Revista Iberoamericana \\ de las Ciencias Sociales y \\ Humanísticas}

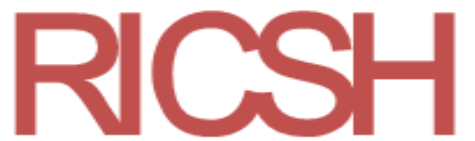

ISSN: $2395-7972$

Gomes, S. J. (2016). Liderança em contextos instáveis: stresse e stressores dos gerentes prisionais e agentes penitenciários das unidades prisionais do Estado da Bahia. (tesis de doctorado). Universidade Autónoma de Lisboa, Lisboa.

Gomes, S. J. e Silva, J. F. (2017). Perspectivas da efetividade da EaD na inclusão de detentos do sistema prisional baiano no ensino superior. UFBA EaD em Revista, 2(1).

Laier, M. G. A. (2016). Atrás das grades: questões de gênero na prisão feminina de João Pessoa. Sociedade em Debate, 22(2), 191-226.

Lambert, E. G., Barton, S. M. and Hogan, N. L. (2014). The Association Between Correctional Orientation and Organizational Citizenship Behaviors Among Correctional Staff. International Journal of Offender Therapy and Comparative Criminology, 58(8).

Lobo, K. D. y Vilarrubia, I. Y. R. (2017). Sistema carcelario Argentina-Brasil desde una perspectiva de género. Criminalidad Femenina. Revista de la Facultad de Derecho y Ciencias Sociales y Políticas, 11(20), 217-238.

Ministério da Justiça e da Segurança Pública (2016). Encuesta Nacional de Información Penitenciaria, Infopen Mujeres. Brasília, Brasil: Ministério da Justiça e da Segurança Pública. Recuperado de http://depen.gov.br/DEPEN/depen/sisdepen/infopenmulheres/infopenmulheres_arte_07-03-18.pdf.

Ordem dos Advogados do Brasil [OAB]. (2016). Relatório Visita Aos Presídios. Salvador, Brasil: Comissão Especial de Sistema Prisional e Segurança Pública do Conselho Regional da Ordem dos Advogados do Brasil no Estado da Bahia. Recuperado de http://gestor.oabba.org.br/fileadmin/user_upload/Transparencia/Comissao_Especial_de_Sistema_Prisional _e_Seguranca_Publica/relatoriovisitapresidios_web.pdf.

Pekny, A. C. y de Mattos, C. (2017). Principales retos de la violencia y la criminalidad en Brasil. Bogotá, Colombia: FES Seguridad. Recuperado de https://library.fes.de/pdffiles/bueros/la-seguridad/13967.pdf.

Santoro, A. E. R., Pereira, A. C. A. e de Lara, M. B. (2018). Gênero e prisão: O encarceramento de mulheres no sistema penitenciário brasileiro pelo crime de tráfico de drogas. Meritum, Revista de Direito da Universidade FUMEC, 13(1), 87-112.

Secretaria de Administração Penitenciária e Ressocialização [SEAP]. (2018). Plano Estadual de Atenção à Mulher Privada de Liberdade e Egressas do Sistema Prisional 2018-2019. Salvador, Brasil: Governo do Estado da Bahia. Recuperado de 


\section{Revista Iberoamericana \\ de las Ciencias Sociales y \\ Humanísticas}

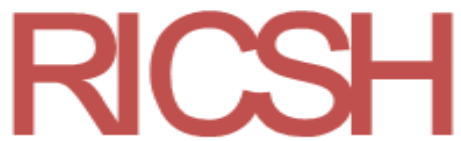

ISSN: $2395-7972$

http://depen.gov.br/DEPEN/dirpp/cgpc/politica-para-mulheresepromocao-dasdiversidades/promocao-das-mulheres BAHIA__ABRIL2018.pdf.

Serrano, F. P. (2016). El enfoque diferencial de género en las intervenciones socioeducativas en ámbitos penitenciarios. RES: Revista de Educación Social, 22(1), 109-121.

Sindicatos dos Servidores Penitenciários do Estado da Bahia [Sinspeb]. (2018). Relatório Sobre o Conjunto Penal de Feira de Santana. Salvador, Brasil: Sindicatos dos Servidores Penitenciários do Estado da Bahia. Recuperado de http://sinspeb.org.br/informativo/wpcontent/uploads/2019/01/Relatorio-sobre-oCPFS.pdf.

Sindicatos dos Servidores Penitenciários do Estado da Bahia [Sinspeb]. (2019). Relatório Sobre o Conjunto Penal de Paulo Afonso. Salvador, Brasil: Sindicatos dos Servidores Penitenciários do Estado da Bahia. Recuperado de_http://sinspeb.org.br/informativo/wpcontent/uploads/2019/02/Relatorio-sobre-o-CPPA.pdf.

Zamboni, M. (2017). O barraco das monas na cadeia das coisas: notas etnográficas sobre a diversidade sexual e de gênero no sistema penitenciário. ARACE-Direitos Humanos em Revista, 4(5), 93-115. 